\title{
Increased expression of KPNA2 predicts unfavorable prognosis in ovarian cancer patients, possibly by targeting KIF4A signaling
}

Xiangrong Cui ${ }^{1}$, Honghong Wang ${ }^{2}$, Xueqing $\mathrm{Wu}^{1}$, Kai Huo ${ }^{3}$ and Xuan $\mathrm{Jing}^{4^{*}}$

\begin{abstract}
Background: Karyopherin a-2 (KPNA2) is a member of karyopherin family, which is proved to be responsible for the import or export of cargo proteins. Studies have determined that KPNA2 is associated with the development and prognosis of various cancers, yet the role of KPNA2 in ovarian carcinoma and its potential molecular mechanisms remains unclear.
\end{abstract}

Materials and methods: The expression and prognosis of KPNA2 in ovarian cancer was investigated using GEPIA and Oncomine analyses. Mutations of KPNA2 in ovarian cancer were analyzed by cBioPortal database. The prognostic value of KPNA2 expression was evaluated by our own ovarian carcinoma samples using RT-qPCR. Subsequently, the cell growth, migration and invasion of ovarian cancer cells were investigated by CCK-8 and transwell assay, respectively. The protein levels of KPNA2 and KIF4A were determined by western blot.

Results: We obtained the following important results. (1) KPNA2 and KIF4A wereoverexpressed in ovairan cancer tissues and cells. (2) Among patients with ovarian cancer, overexpressed KPNA2 was associated with lower survival rate. (3) Mutations (R197* and S140F) in KPNA2 will have some influences on protein structure, and then may cause protein function abnormal. (4) KPNA2 konckdown inhibited proliferation, migration, invasion, as well as the expression of KIF4A.

Conclusion: KPNA2, as a tumorigenic gene in ovarian cancer, accelerated tumor progression by up-regulating KIF4A, suggesting that KPNA2 might be a hopeful indicator of treatment and poor prognosis.

Keywords: KPNA2, Ovarian cancer, Prognosis, KIF4A

\section{Introduction}

Ovarian cancer (OC), a common gynecological malignancy, is the fifth leading cause of death among women around the world [10, 28]. According to statistics published in National comprehensive Cancer Network $(\mathrm{NCCN})$, more than half of patients can not live more

\footnotetext{
* Correspondence: jx05070103@163.com

${ }^{4}$ Clinical Laboratory, Shanxi Prov. People's Hospital, Affiliated of Shanxi Medical University, Taiyuan 030001, China

Full list of author information is available at the end of the article
}

than 5 years after diagnosis [1]. A number of genetic alterations associated with development and progression of ovarian cancer have been reported; however, most patients are in the advanced stage at the time of initial diagnosis $[13,21,31]$. It is urgent to reveal the pathophysiological mechanism of ovarian cancer, optimize the treatment plan, and realize the early diagnosis of ovarian.

Karyopherin $\alpha-2$ (KPNA2) is a member of karyopherin $\alpha$ family (KPNA), which plays an important role in

(c) The Author(s). 2021 Open Access This article is licensed under a Creative Commons Attribution 4.0 International License, which permits use, sharing, adaptation, distribution and reproduction in any medium or format, as long as you give appropriate credit to the original author(s) and the source, provide a link to the Creative Commons licence, and indicate if changes were made. The images or other third party material in this article are included in the article's Creative Commons licence, unless indicated otherwise in a credit line to the material. If material is not included in the article's Creative Commons licence and your intended use is not permitted by statutory regulation or exceeds the permitted use, you will need to obtain permission directly from the copyright holder. To view a copy of this licence, visit http://creativecommons.org/licenses/by/4.0/ The Creative Commons Public Domain Dedication waiver (http://creativecommons.org/publicdomain/zero/1.0/) applies to the data made available in this article, unless otherwise stated in a credit line to the data. 
nucleocytoplasmic transport [3, 18, 29]. Studies have determined that KPNA2 is associated with modulating both the nuclear import and the cytoplasm export of molecules $[4,9,20]$. Recently, higher expression of KPNA2 was demonstrated in breast cancer [8], burkitt lymphoma [23], melanoma [33], bladder cancer [25], and correlated with the poor prognosis of patients. However, the exact role of KPNA2 in ovarian cancer and its underlying molecular mechanism have not been elucidated.

In current research, we hypothesized that KPNA2 may be a promising candidate for the potential diagnostic and prognosticof ovarian cancer. To confirm this hypothesis, we used bioinformatics methods to analyze the expression and prognostic value of KPNA2 in ovarian cancer. Furthermore, we used ovarian cancer cell lines to identify the molecular mechanisms underlying the effect of overpressed KPNA2 on ovarian carcinoma. Our results determined that KPNA2 is overexpressed in ovarian carcinoma and plays an important role in malignant transformation through regulating KIF4A signaling pathway. These observational findingswill contribute to the development and optimization of novel insights into diagnosis and prognosis for ovarian carcinoma.

\section{Materials and methods \\ Clinical samples}

This study was performed on 35 patients with ovarian carcinoma from Shanxi province of China. Frozen tissues were collected from the Department of Gynaecology and Obstetrics from Tumor Hospital of Shanxi (China). The average age of the patients was 60.14 years (23-77 years). The study was performed following the Declaration of Helsinki set of principles and approved by the Ethics Committee of the Shanxi Medical University (Ethical code: 201922021). Informed written consent was obtained from all patients enrolled.

\section{Cell culture and transfections}

The human ovarian carcinoma SKOV3 cells were purchased from American Type Culture Collection. Cells were cultured in DMEM/F12 medium supplemented with 10\% FBS (Gibco, Thermo Fisher Scientific, Inc.) in a humidified atmosphere with $5 \% \mathrm{CO}_{2}$ at $37^{\circ} \mathrm{C}$. siRNA targeting KPNA2 (si-KPNA2) and siRNA negative control (si-NC) were constructed and purchased from GenePharma (Shanghai, China). Cell transfection was performed using the Lipofectamine 20,000 (Invitrogen, Carlsbad, CA, USA) according to the manufacturer's instructions for $48 \mathrm{~h}$.

\section{Gene expression profiling interactive analysis}

Gene Expression Profiling Interactive Analysis (GEPIA) database (http://gepia.cancer-pku.cn/) was utilized to analyze the RNA sequencing expression of 9736 tumors and 8587 normal samples from The Cancer Genome Atlas (TCGA) and Genotype-Tissue Expression (GTEx) project data [26]. In GEPIA, KPNA2 expression various human cancers and adjacent normal tissues was obtain, furthermore the KPNA2 expression in ovarian carcinoma and corresponding normal tissues was validated.

\section{Oncomine database analysis}

KPNA2 gene expression levels in malignancies were analyzed from Oncomine database, a network based data mining platform including 715 datasets and 86,733 samples (http://www.oncomine.org) [24]. Paired Student's ttest was performed to compare group means. A foldchange of at least 2 with a $P$-value $<0.0001$ was defined as clinically significant, as previously described.

\section{cBioportal database analysis}

The term "KPNA2" was adopt to search the cBio Cancer Genomics Portal (cBioPortal) database (http://www. cbioportal.org/) and ovarian serous cystadenocarcinoma (TCGA, Nature 2011, $n=489$ ) cohort was performed [6, 11]. Mutations, putative copy number aberrations, and co-expression from RNA-seq data, were assessed as the search parameters to visualize the results.

\section{University of California Santa Cruz Cancer Genomics Browser Analysis}

The University of California Santa Cruz (UCSC) Xena browser (http://xena.ucsc.edu/), included 758 cases of ovarian cancer with genomic and clinical, was performed to access TCGA ovarian cancer data. The relationship between the transcriptional expression of KPNA2 and KIF4 was accessed through ovarian carcinoma cohort in TCGA database (TCGA-Ovarian Cancer) [12].

\section{UALCAN analysis}

UALCAN (http://ualcan.path.uab.edu), an interactive web resource, is performed to analyze relative mRNA expression of potential genes (TCGA and MET500 transcriptome sequencing) and relationship of the mRNA expression with various tumor subtypes, including age, gender, tumor stages, and other clinicopathological features [7]. In our research, UALCAN was utilized to access the mRNA expression of KPNA2 in primary ovarian carcinoma tissues and its association with various tumor molecular subtypes and clinicopathologic parameters. 


\section{PrognoScan online platform}

PrognoScan online platform (http://www.prognoscan. org) was utilized to access the prognostic value of KPNA2 expression in ovarian carcinoma patients [19]. In this web based online platform, the $P$-value, HR and $95 \%$ confidence intervals (CI) based on a KPNA2 expression was automatically calculated by PrognoScan.

\section{Kaplan-Meier plotter analysis}

The Kaplan Meier plotter (http://kmplot.com/analysis/) is capable to evaluate the role of $54 \mathrm{k}$ genes on prognosis in various cancer types from the databases containing GEO, EGA, and TCGA. We accessed the association between the KPNA2 expression and prognosis using the ovarian cancer cohort [22].

\section{CCK-8 detection of viability}

Cell Counting Kit-8 (CCK-8, ATgene, Taiwan, China) was performed to evaluate the cell viability. Cells were tiled into 96-well plates at $5 \times 10^{3}$ cells per well and subjected in $100 \mu \mathrm{l}$ serum-free medium for $24,48,72$, and $96 \mathrm{~h}$. Then, $10 \%$ CCK-8 solution was added to each well for $2 \mathrm{~h}$ and the absorbance (A) valuewas detected at 450 $\mathrm{nm}$ by microplate reader (BioTek, Epoch, VT). All tests were repeated eight times, and each experiment was carried out at least three replicate.

\section{Cell migration and invasion ability assay}

Invasion and migration abilities of ovarian cancer cells were determined using Boyden assay. $600 \mu \mathrm{l}$ DMEM/F12 supplemented with $10 \%$ FBS was added to the 24-well plate, followed by Transwell chamber (Millipore, Burlington, MA, USA) seeded with $4 \times 10^{4}$ cells. For cell invasion assay, $100 \mu \mathrm{g} / \mathrm{ml}$ Matrigel (BD Biosciences, Boston, MA, USA) was added to the upper layer of chamber. After incubation for $24 \mathrm{~h}$ at $37^{\circ} \mathrm{C}$, the cells in the upper chamber were removed. Cells were fixed with $600 \mu \mathrm{l}$ fixative solution for $10 \mathrm{~min}$. The chamber was stained with $600 \mu \mathrm{l}$ crystal violet for $15 \mathrm{~min}$. Finally, an inverted microscope (Eclipse Ti2; Nikon Corporation) was used to photograph and calculate the invading and migratory cells.

\section{Quantitative real-time PCR (qRT-PCR)}

RNA extraction kit was performed to extract the total RNAs from tissues and cells. RNA was reverse transcribed into complementary DNA by Prime Script ${ }^{\text {ta }}$ RT Master Mix kit. qRT-PCR was performed using SYBR $^{\circ}$ Premix Ex Taq Kit. For PCR amplification, GAPDH was presented as an internal reference. The primer sequences were as follows: KPNA2, 5'-ATTGCAGGTGATGGCT CAGT-3' (forward) and 5'-CTGCTCAACAGCATCTAT CG-3' (reverse); GAPDH, 5'-ACCACAGTCCATGCCA TCAC-3' (forward); and 5'-TCCACCA CCCTGTTGCT GTA-3' (reverse). The method of $2^{-\Delta \Delta C t}$ was performed to calculate the relative gene expression.

\section{Western blot analysis}

Proteins from ovarian carcinoma cell lines were extracted with lysis buffer follow instructions. The BCA method was

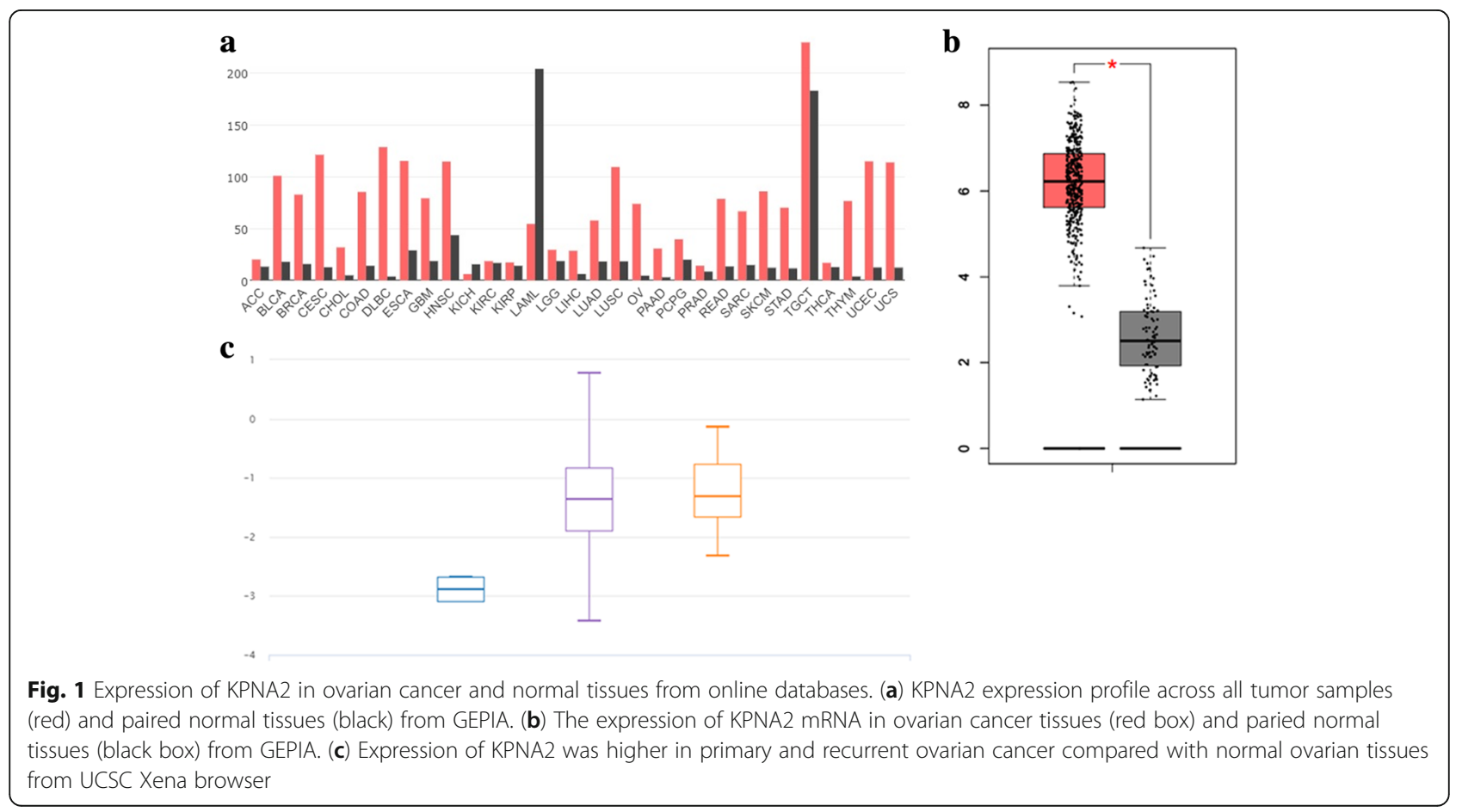




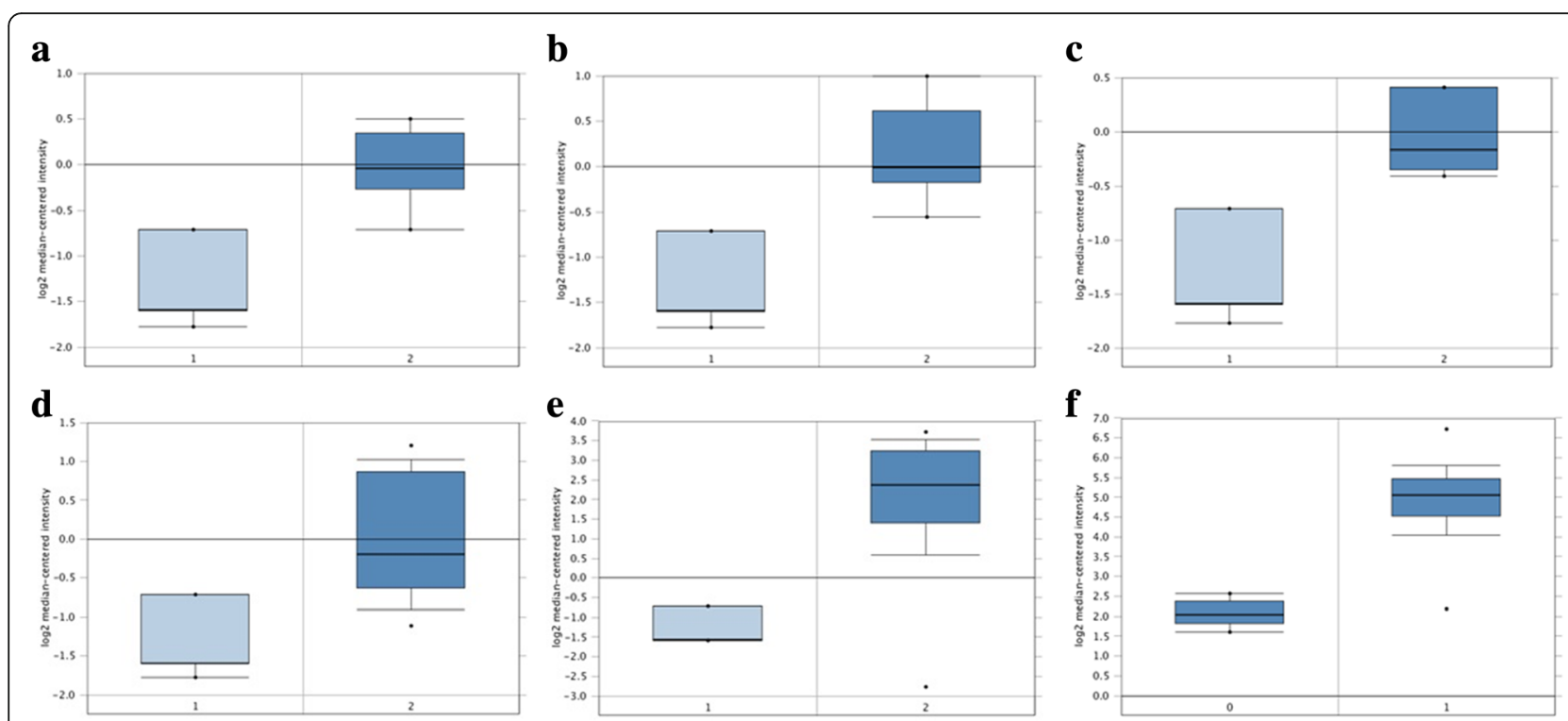

Fig. 2 KPNA2 analysis in ovarian cancer (Oncomine database). The box plot compares KPNA2 expression in cancer samples (right) and matches normal (left) samples generated from the Oncomine database. (a) ovarian mucinous adenocarcinoma; (b) ovarian endometrioid adenocarcinoma; (c) ovarian clear cell adenocarcinoma; (d) ovarian serous adenocarcinoma; (e) ovarian serous surface papillary carcinoma, (f) ovarian

serous cystadenocarcinoma

performed to determine protein concentration using BCA Protein Assay kit. Protein lysate was subjected to SDSPAGE gels and transferred subsequently onto PVDF membranes. The membrane was blocked by TBS containing $0.1 \%$ Tween-20. Then, membranes were incubated with rabbit anti KPNA2 monoclonal antibody (ab170495; 1:1000; Abcam, Cambridge, MA, USA), KIF4A (ab124903; 1:1000; Abcam, Cambridge, MA, USA) or GAPDH (cat. no. T0004; 1:5000; Affinity Biosciences) at $4{ }^{\circ} \mathrm{C}$ overnight, followed by goat anti-rabbit IgG-HRP secondary antibodies for $2 \mathrm{~h}$ at $37^{\circ} \mathrm{C}$. ECL reagents were performed to visualize protein bands.

\section{Statistical analysis}

GraphPad Prism 7.0 was performed to statistical analysis. Student's t-test was adopt to analysis differences between two groups. Overall survival was calculated by Kaplan-Meier survival curves and the statistical comparisons were analyzed by Log-rank test. $P$-value $<0.05$ is applied as the threshold.

\section{Results}

\section{KPNA2 transcript expression status in human ovarian}

\section{Cancer}

The expression profile of KPNA2 was identified by GEPI A. The data revealed that KPNA2 was significantly higher in BLCA, BRCA, CESC, COAD, DLBC, ESCA, GBM, HNSC, LIHC, LUAD, LUSC, OV, PAAD, READ, SKCM, STAD, THYM, UCEC, UCS (Fig. 1a and b). To further confirm this result, the UCSC Xena browser was carried out to determine the expression profile of KPNA2, which was higher expression in primary and recurrent ovarian carcinoma compared with normal ovarian tissues (Fig. 1c). Then, the Oncomine database was adopted to evaluate KPNA2 expression in different histologic subtypes of primary ovarian cancers. KPNA2 expression was significantly higher in ovarian mucinous adenocarcinoma, ovarian endometrioid adenocarcinoma, ovarian clear cell adenocarcinoma, ovarian serous adenocarcinoma, ovarian serous surface papillary carcinoma, ovarian serous cystadenocarcinoma (Fig. 2 and Table 1).

Table 1 KPNA2 expression in ovarian cancer

\begin{tabular}{lllllll}
\hline Cancer subtype & $\boldsymbol{p}$-value & Fold change & $\boldsymbol{t}$-test & Rank (\%) & Sample & Reference \\
\hline Ovarian Mucinous Adenocarcinoma & $2.02 \mathrm{E}-4$ & 2.523 & 5.742 & 1 & 9 & 8 \\
Ovarian Endometrioid Adenocarcinoma & $6.60 \mathrm{E}-5$ & 2.891 & 6.067 & 1 & 9 & 8 \\
Ovarian Clear Cell Adenocarcinoma & $2.30 \mathrm{E}-4$ & 2.554 & 5.987 & 1 & 7 & 8 \\
Ovarian Serous Adenocarcinoma & $1.07 \mathrm{E}-4$ & 2.544 & 5.330 & 4 & 20 & 8 \\
Ovarian Serous Surface Papillary Carcinoma & $1.57 \mathrm{E}-8$ & 11.343 & 10.339 & 2 & 28 & 8 \\
Ovarian Serous Cystadenocarcinoma & $3.72 \mathrm{E}-9$ & 7.566 & 24.875 & 1 & 586 & TCGA (No Associated Paper) \\
\hline
\end{tabular}


$\mathbf{a}_{40}$

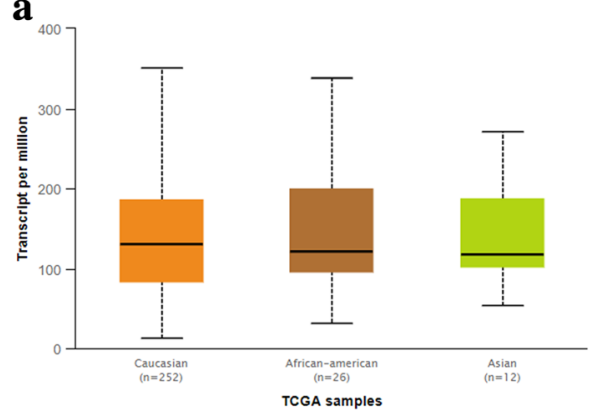

c

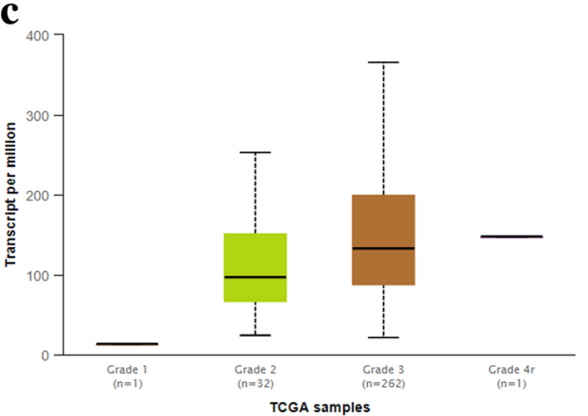

b

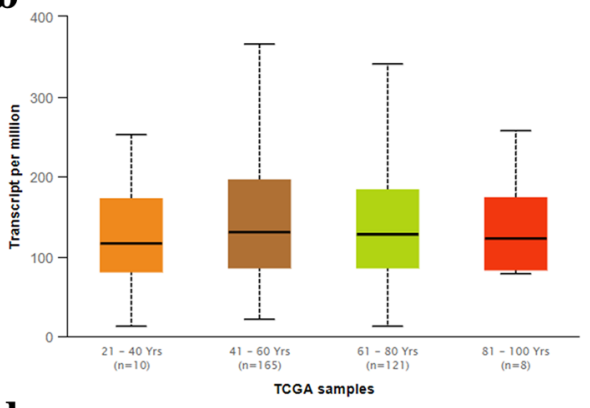

d

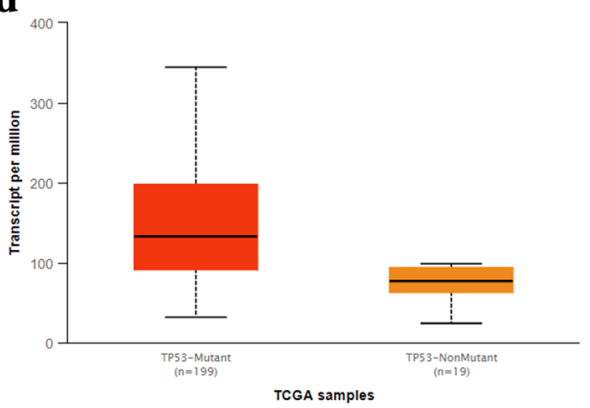

Fig. 3 Genetic alterations of KPNA2 in ovarian cancer subgroups. Subgroup analysis of multiple clinic pathologic features of ovarian cancer samples in the TCGA. (a) rases; (b) ages; (c) stages; (d) TP53-Mutant

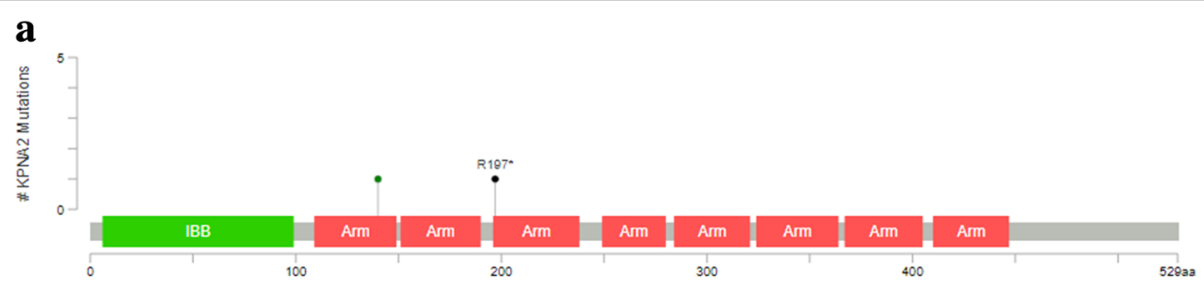

b

\begin{tabular}{|c|c|c|c|c|}
\hline \multicolumn{4}{|c|}{ Mutation s of KPNA2 in } & ovarian cancer (TCGA) \\
\hline Sample ID & Protein Change & Mutation Type & Copy\# & $\begin{array}{l}\text { \#Mut in } \\
\text { Sample }\end{array}$ \\
\hline TCGA-29-1777-01 & $R_{197^{*}}$ & Nonsense & Diploid & 126 \\
\hline TCGA-23-1116-01 & S14OF & Missense & Gain & 183 \\
\hline
\end{tabular}

C

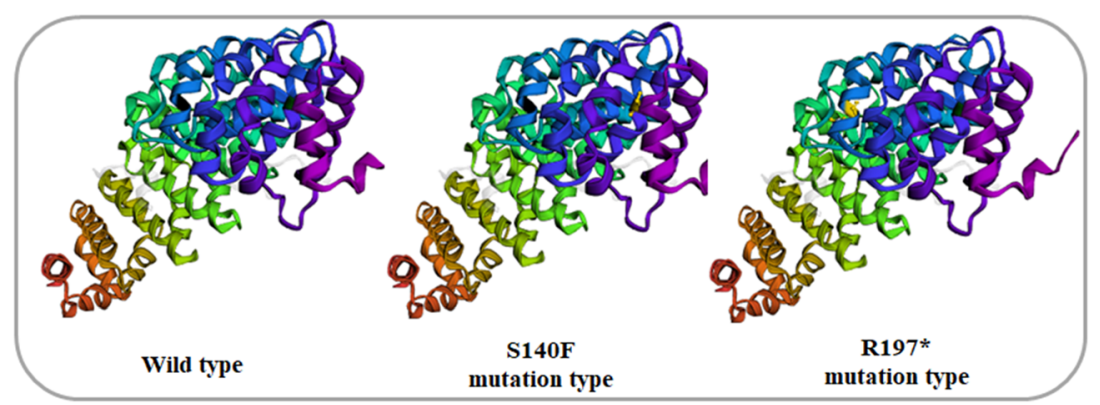

Fig. 4 KPNA2 Mutations in Ovarian Cancer. (a) The graphical view shows the Pfam protein domains and the positions of specific mutations. The length of the line connecting the mutation annotation to the protein is indicative of the number of samples that have the mutation. The most recurrent mutations are labeled in the graphical view. (b) The tabular view provides additional information about all mutations in each query gene. (c) 3D protein structure visualization and analysis were performed 


\section{Genetic alterations in KPNA2 and Clinicopathological parameters}

Further subgroup analysis according multiple clinic pathologic features of ovarian carcinoma in the TCGA was shown in Fig. 3. The results determined that KPNA2 expression level was not significantly different among different races and age groups (Fig. $3 \mathrm{a}$ and b). In addition, the expression level of KPNA2 was significantly associated with the stages of ovarian cancer (Fig. 3c). A significant overexpression of KPNA2 in TP53-Mutant samples than TP53-NonMutant samples of ovarian cancer in subgroup analyses based on whether TP53 is mutated (Fig. 3d).

\section{KPNA2 mutations in ovarian Cancer}

Mutations in ovarian cancer mainly occur in Arm domain, which analyzed by cBioPortal database. As shown in Fig. 4, the graphical view reveals the KPNA2 protein domains and the positions of specific mutations. The most recurrent mutations are labeled in the graphical view (Fig. 4a). Furthermore, the additional information about all mutations (R197* and $S 140 F)$ in KPNA2 was revealed in tabular view (Fig. 4b). 3D protein structure visualization and analysis were performed using cBioPortal database. As shown in the Fig. 4c, Ser140 was replaced by Phe, while the R197 was replaced by termination codon. Therefore, the R197* and S140F mutations will have some influences on protein structure, and then may cause protein function abnormal.

\section{Relationship of KPNA2 expression and prognosis in ovarian cancer}

To evaluate whether KPNA2 expression level has predictive significance for ovarian carcinoma prognosis, we detected the expression of KPNA2 mRNA and its relationship with prognosis. Our own results found the upregulation of KPNA2 mRNA expression and worse probabilities of survival in ovarian carcinoma (Fig. 5a and b). Furthermore, we used PrognoScan Online Platform and Kaplan-Meier Plotter Analysis to further confirm the predictive value of KPNA2 expression for ovarian prognosis. Both databases verified the KPNA2 expression was negatively related to overall survival (PrognoScan Online Platform: $\mathrm{HR}=1.45$ [1.00-2.11], $p=0.049$; Kaplan-Meier Plotter Analysis: $\mathrm{HR}=1.15$ [1.00-1.31], $\quad p=0.044$ ) (Fig. 5c and d).

\section{Co-expression of KPNA2 gene}

To further determine the regulatory mechanisms underlying the role of KPNA2 in ovarian carcinoma, data

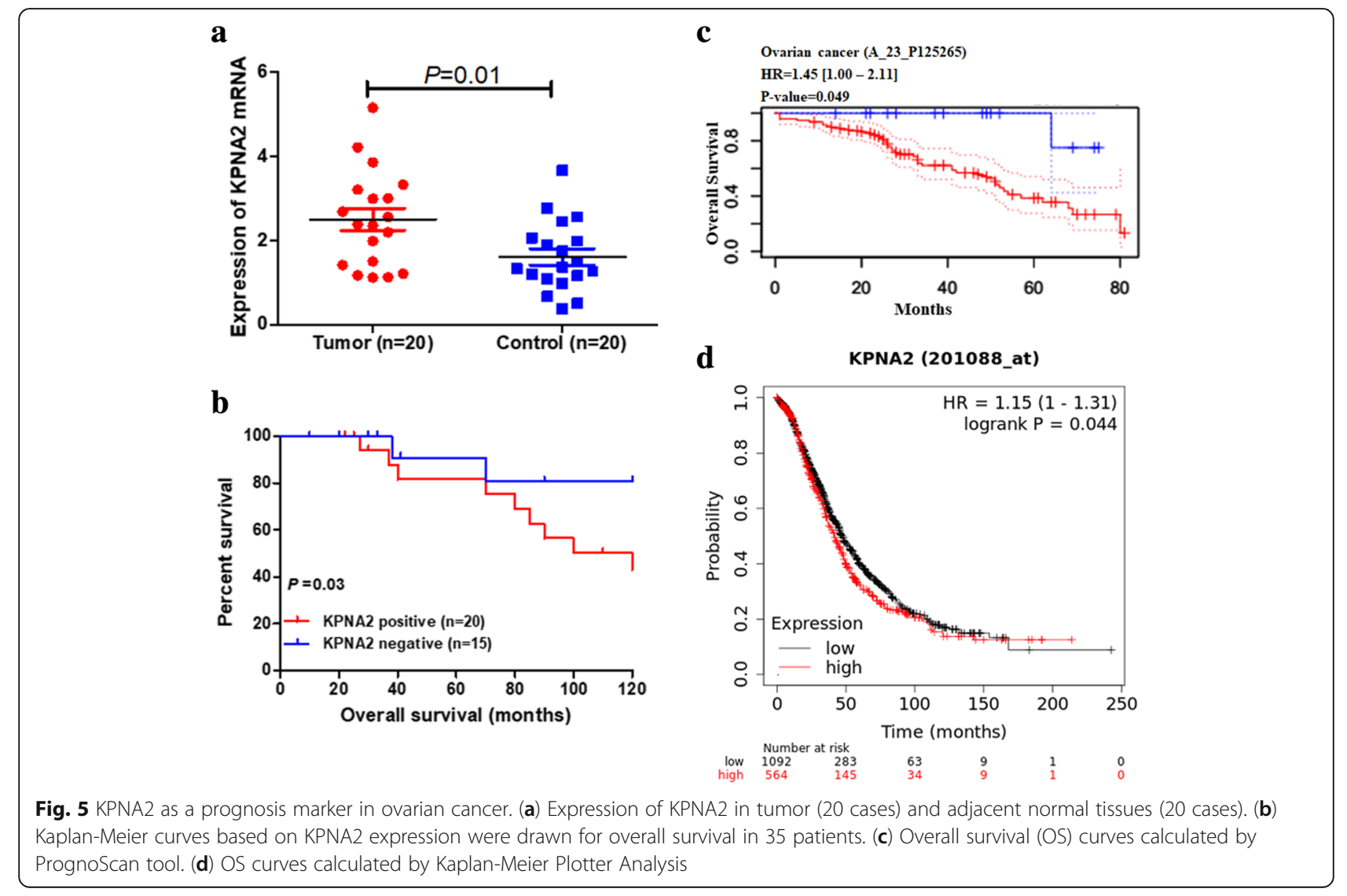


mining was performed on a ovarian carcinoma cohort using cBioPortal. Kinesin family member 4A (KIF4A) is a highly related gene (Fig. 6a); it promoted progression in various tumor tissues by regulating chromosome segregation machinery in mediating spindle organization and cytokinesis [2, 5, 32]. A regression analysis using cBioportal showed that KPNA2 and KIF4A levels are highly correlated (Pearson's correlation $=0.69$; Spearman's correlation $=0.69$ ) (Fig. $6 \mathrm{~b}$ ). The positive correction between KPNA2 and KIF4A mRNA expression was determined using data from GEPIA (Fig. 6c). By investigating ovarian carcinoma data in TCGA using UCSC Xena, the positive correlation was further confirmed (Fig. 6d and e). These data informed that KPNA2 could be associated with the KIF4A pathway in ovarian carcinoma. To investigate the genetic alterations of KIF4A, the GEPI tool was applied to determine KIF4A expression profiles. The results of KIF4A analysis informed that KIF4A highly expressed in ovarian carcinoma tissues compared with normal ovarian tissues (Fig. 7a). Then, our own results further confirmed the overexpression of KIF4A mRNA and worse probabilities of survival in ovarian carcinoma (Fig. 7b).

\section{KPNA2 accelerated the biological characteristics of ovarian Cancer cells through up-regulating KIF4A}

To access the potential role of KPNA2 in ovarian cancer cells, the endogenous expression level of KPNA2 was knocked down with si-KPNA2 transfection. As exhibited in Fig. 8a, the KPNA2 expression was significantly reduced in ovarian cancer cells transfected with si-KPNA2. Next, CCK-8 assay demonstrated that KPNA2 knockdown significantly weakened the ability of proliferation of ovarian carcinoma cells (Fig. 8b). Furthermore, $\mathbf{a}$

\begin{tabular}{|c|c|c|c|c|}
\hline $\begin{array}{l}\text { Top15 genes } \\
\text { Correlated Gene }\end{array}$ & $\begin{array}{l}\text { ositively } \\
\text { cytoband }\end{array}$ & $\begin{array}{l}\text { ated to } \mathbf{K} \\
\text { Spearman's } \\
\text { correlation }\end{array}$ & $\begin{array}{c}\text { NA2 ex } \\
\text { p-Value }\end{array}$ & $\begin{array}{r}\text { ression } \\
q \text {-value }\end{array}$ \\
\hline KIF4A & Xq13.1 & 0.689 & $9.58 \mathrm{e}-46$ & $1.77 e-41$ \\
\hline BIRC5 & $17 \mathrm{q} 25.3$ & 0.680 & $3.25 \mathrm{e}-44$ & $3.01 \mathrm{e}-40$ \\
\hline CENPE & $4 q 24$ & 0.677 & $1.01 \mathrm{e}-43$ & $6.200-40$ \\
\hline CCNB2 & $15 q 22.2$ & 0.673 & $5.44 \mathrm{e}-43$ & $2.52 \mathrm{e}-39$ \\
\hline PSMD12 & $17 q 24.2$ & 0.658 & $1.61 \mathrm{e}-40$ & $5.94 \mathrm{e}-37$ \\
\hline CCNA2 & $4 \mathrm{q} 27$ & 0.657 & 2.12e- 40 & $6.55 \mathrm{e}-37$ \\
\hline RACGAP1 & $12 q 13.12$ & 0.650 & $2.47 \mathrm{e}-39$ & $6.54 \mathrm{e}-36$ \\
\hline KIF23 & $15 q 23$ & 0.649 & $3.35 \mathrm{e}-39$ & $7.75 e-36$ \\
\hline CENPA & $2 \mathrm{p} 23.3$ & 0.647 & $6.50 \mathrm{e}-39$ & $1.34 \mathrm{e}-35$ \\
\hline KIF $2 \mathrm{C}$ & 1p34.1 & 0.645 & $1.67 \mathrm{e}-38$ & $3.09 \mathrm{e}-35$ \\
\hline RRM2 & $2 \mathrm{p} 25.1$ & 0.643 & $3.04 \mathrm{e}-38$ & $5.12 \mathrm{e}-35$ \\
\hline MELKK & $9 p 13.2$ & 0.642 & $4.05 \mathrm{e}-38$ & $6.25 \mathrm{e}-35$ \\
\hline PRR11 & $17 q 22$ & 0.641 & $6.38 \mathrm{e}-38$ & $9.08 \mathrm{e}-35$ \\
\hline BUB1 & $2 q 13$ & 0.640 & $6.99 \mathrm{e}-38$ & $9.24 \mathrm{e}-35$ \\
\hline DEPDC1 & $1 \mathrm{p} 31.3$ & 0.640 & $9.09 \mathrm{e}-38$ & $1.12 \mathrm{e}-34$ \\
\hline
\end{tabular}

d
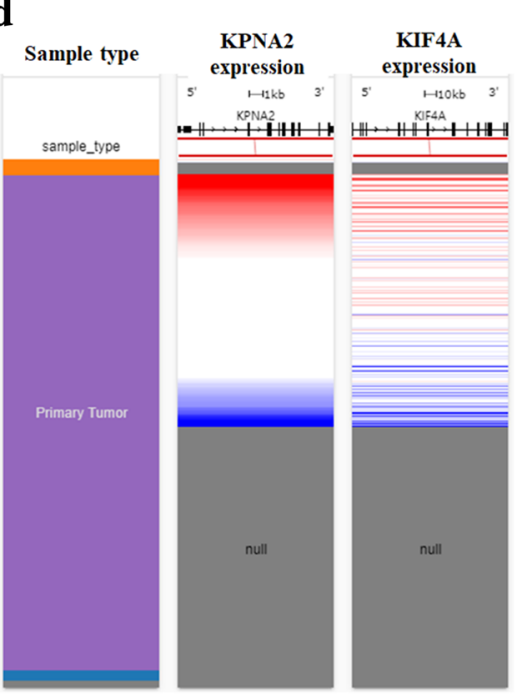

b

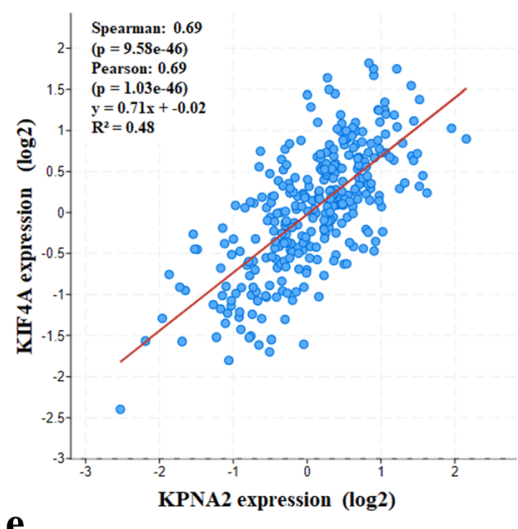

$\mathbf{e}$ c

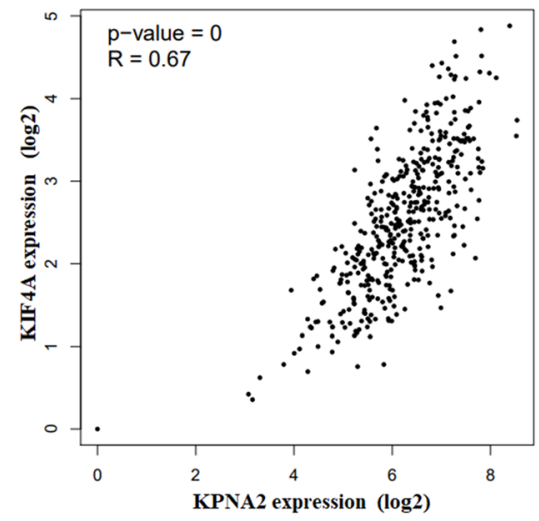

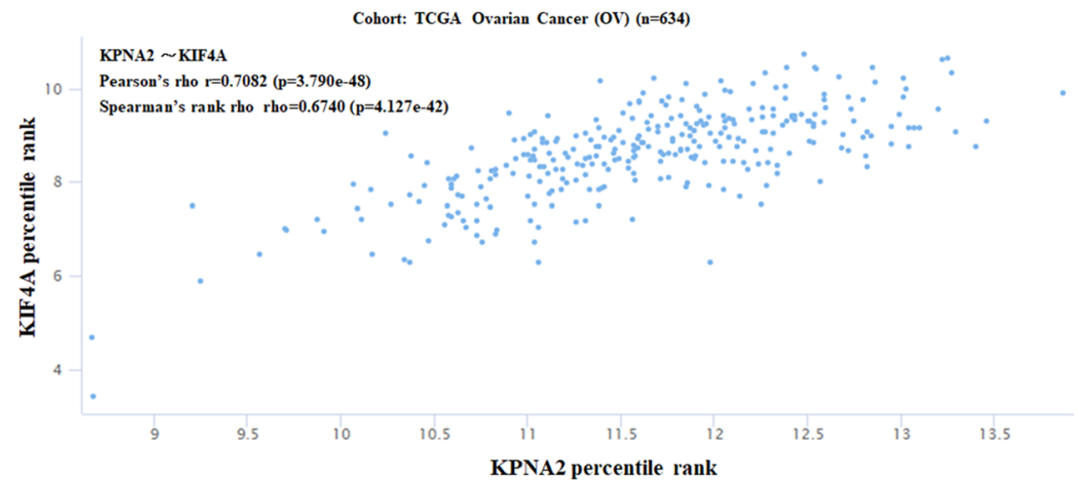

Fig. 6 Co-Expression of KPNA2 gene. (a) Co-expression of KPNA2 gene as determined by cBioPortal. (b) Regression analysis between KPNA2 and KIF4A in ovarian performed by cBioPortal. (c) Relationship between KPNA2 and KIF4A in ovarian cancer through GEPIA. (d) Heat map of KPNA2 and KIF4A mRNA expression in ovarian cancer identified by UCSC Xena. (e) Correlation between KPNA2 and KIF4A mRNA expression in the TCGA database, identified by UCSC Xena 


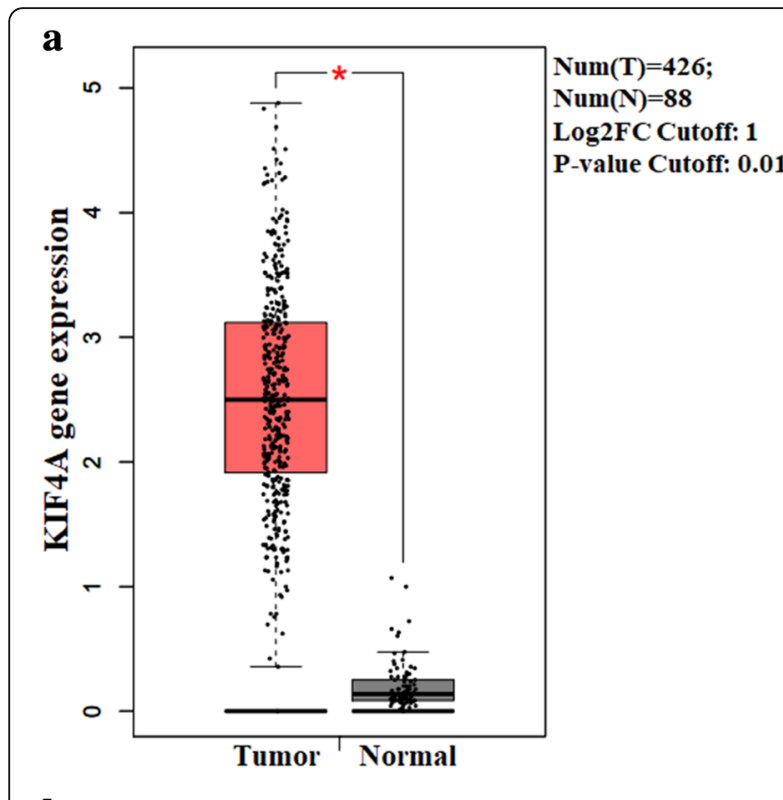

b

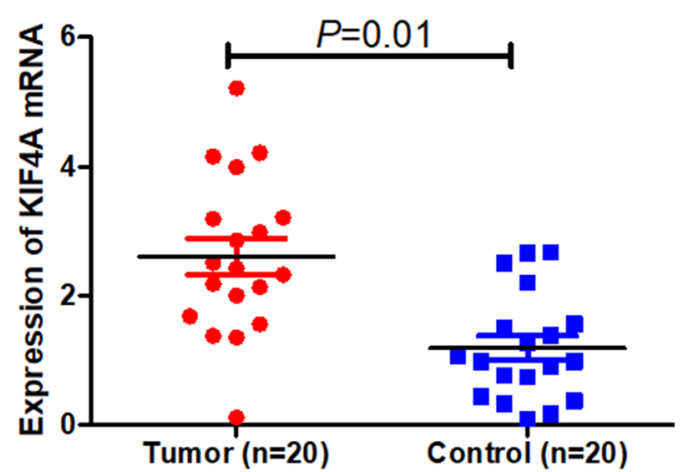

Fig. 7 Expression of KIF4A in ovarian cancer. (a) The expression of KIF4A mRNA in ovarian cancer tissues (red box) and paired normal tissues (black box) from GEPIA. (b) Expression of KIF4A in tumor (20 cases) and adjacent normal tissues (20 cases)

transwell assay revealed that the migratory and invasive cells were collectively declined in ovarian carcinoma cells transfected with si-KPNA2 (Fig. 8c and d). Finally, the expression of KIF4A was inhibited by KPNA2 knockdown of KPNA2 in ovarian carcinoma cells (Fig. 8e and $\mathrm{f}$ ). Above data elucidated that KPNA2 could promote ovarian carcinoma cell progression by upregulating KIF4A in vitro.

\section{Discussion}

KPNA2 is proposed as a crucial adaptor in nucleocytoplasmic transport, which has been suggested to be a transporter of key cell cycle regulators and DNA damage reaction molecules in tumors [3, 17]. Studies have revealed that KPNA2 is abnormally expressed and as a poor prognosis predictor for multiple malignancies, including hepatocellular, bladder and breast carcinoma, and malignant melanoma $[9,16,17,30]$. However, the prognostic value of KPNA2 expression in ovarian carcinoma is still ambiguous.

To explore the effect of KPNA2 in the development, progression, and prognosis of ovarian carcinoma, we detected extensive gene expression profiling with pre-defined parameters in ovarian carcinoma and normal samples. According to GEPIA, KPNA2 was significantly overexpressed in ovarian cancer tissues than in normal controls. Using Oncomine, we found that KPNA2 is significantly upregulated in ovarian mucinous adenocarcinoma, ovarian endometrioid adenocarcinoma, ovarian clear cell adenocarcinoma, ovarian serous adenocarcinoma, ovarian serous surface papillary carcinoma, and ovarian serous cystadenocarcinoma. Furthermore, the mutations of KPNA2 were identified by cBioPortal database. We found that mutations in ovarian cancer mainly occur in Arm domain, and the R197* and S140F mutations might have some influences on protein structure, and then may cause protein function abnormal. Prognosis analysis revealed that the higher expression of KPNA2 correlated significantly with reduced OS. We further adopt our own results validated that overexpression of KPNA2 and worse probabilities of survival in ovarian carcinoma, which determined that KPNA2 mRNA expression may be an independent prognostic biomarker in ovarian carcinoma patients.

By mining co-expression and correlation analysis data, we demonstrated that KPNA2 and KIF4A were both overexpressed in ovarian carcinoma. KIF4A, a member of the kinesin superfamily (KIFs), is associated with multiple cellular activities, particularly spindle formation and centrosome assembly in mitosis, chromosome concentration and separation [5, 27]. Furthermore, multiple studies have demonstrated that KIF4A is closely correlated with the occurrence and development of various malignancies, such as lung adenocarcinoma [34], hepatocellular carcinoma [15], colorectal cancer [14], prostate cancer [5]. Then, we identified the expression of KIF4A in ovarian carcinoma through GEPIA database and our own samples. The results determined that KIF4A mRNA expression was significantly up-regulated in ovarian carcinoma samples compared with controls. These results illustrate that the expression of KPNA2 may regulate tumor invasion and metastasis correlated with KIF4A transcription.

Then, we further validated the function of KPNA2 in growth and metastasis of ovarian carcinoma cells through knockdown the expression of KPNA2 using siRNA technology. The experiment demonstrates that KPNA2 might be a potent factor promoting the proliferation, migration, and invasion of ovarian carcinoma. 

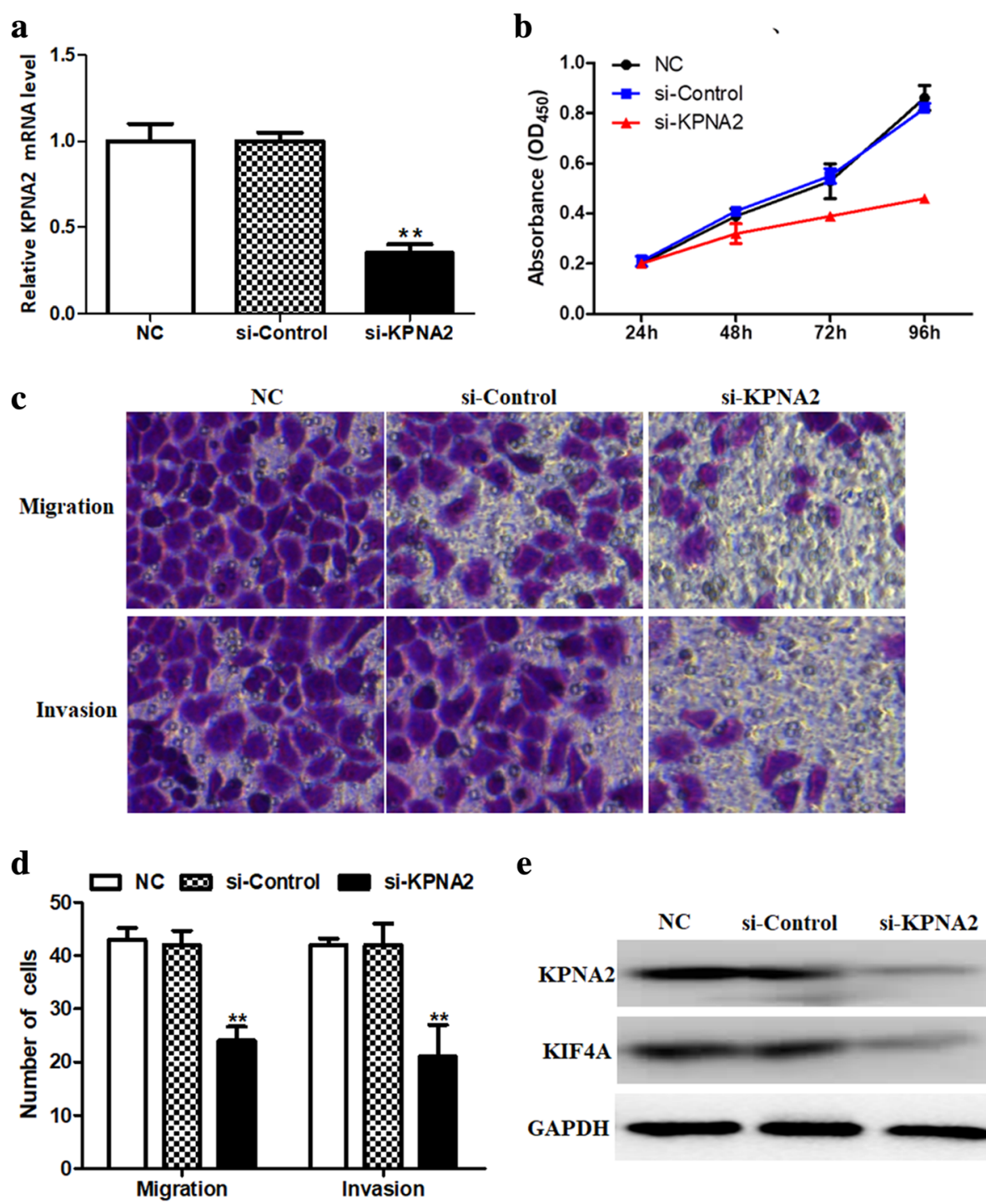

$\mathbf{e}$

\section{$\mathbf{f}$}
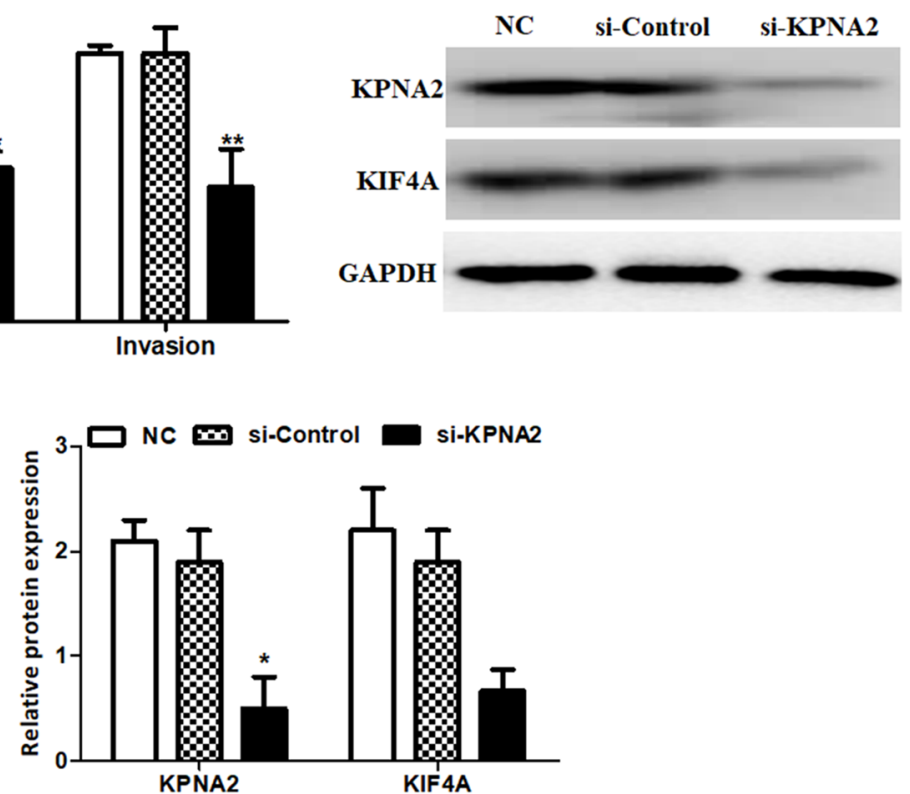

Fig. 8 KPNA2 accelerated the cell growth, invasion, and migration in ovarian cancer through KIF4A. (a) The expression of KPNA2 was prominently reduced in ovarian cancer cells transfected with si-KPNA2. (b) The cell proliferation of ovarian cancer cell lines transfected with different interference sequences, cell proliferation was the weakest in si-KPNA2 group. (c and $\mathbf{d}$ ) Invasion and migration assays of two kinds of cancer cells under different interference conditions. (e and $\mathbf{f}$ ) The western blot showed KIF4A expression was significantly decreased in si-KPNA2 group ovarian cells. ${ }^{*} P<0.05$ and ${ }^{*} P<0.01$, compared with $N C$ group

Moreover, we identified the expression level of KIF4A after KPNA2 knockdown in ovarian cancer cell lines. As anticipated, we found that the down-regulation of of
KIF4A expression after KPNA2 knockdown in ovarian cancer cell lines. These experiments preliminarily validate that KPNA2 exerted its effects on the malignant 
behaviors of ovarian cancer cells through the KIF4A signaling pathway.

\section{Conclusion}

In general, the present study confirm that KPNA2 is highly expressed in ovarian carcinoma, as well as an independent risk factor for poor prognosis in patients with ovarian carcinoma. Also, we elucidate that KPNA2 has a role in malignancy mainly through the KIF4A signaling, which is a potential target for treating ovarian cancer. However, our understanding of the regulatory mechanism of KPNA2 in ovarian carcinoma is limited and requires further exploration.

\section{Acknowledgements}

Not applicable.

\section{Authors' contributions}

All authors contributed to data analysis, drafting or revising the article, gave final approval of the version to be published, and agree to be accountable for all aspects of the work.

\section{Funding}

This study was supported by the Scientifc Research Project of Shanxi Provincial Department of Health (grant no. 201601070), Initial Scientifc Research Fund of PhD in Shanxi Provincial People's Hospital (grant no. b201635), Natural Science Foundation of Shanxi (grant no. 201901D211519), Natural Science Foundation of Shanxi (grant no. 201901D211546), Research Project Supported by Shanxi Scholarship Council of China (grant no. HGKY2019092), and China Postdoctoral Science Foundation (grant no. 2020 M670703), Fund Program for the Scientific Activities of Selected Returned Overseas Professionals in Shanxi Province (20200033).

\section{Availability of data and materials}

The analyzed data sets generated during the study are available from the corresponding author on reasonable request.

\section{Declarations}

\section{Ethics approval and consent to participate}

Informed consent was obtained from each participant. The entire experiments have already obtained the approval of the Ethics Committee of Shanxi Medical University (Ethical code: 201922021).

\section{Consent for publication}

Not applicable.

\section{Competing interests}

The authors declare that they have no competing interests.

\begin{abstract}
Author details
${ }^{1}$ Reproductive Medicine Center, Children's Hospital of Shanxi and Women Health Center of Shanxi, Affiliated of Shanxi Medical University, Taiyuan 030001, China. ${ }^{2}$ Gynaecology and Obstetrics Department, Children's Hospital of Shanxi and Women Health Center of Shanxi, Affiliated of Shanxi Medical University, Taiyuan 030001, China. ${ }^{3}$ Breast Surgery Department, Tumor Hospital of Shanxi, Affiliated of Shanxi Medical University, Taiyuan 030000, China. ${ }^{4}$ Clinical Laboratory, Shanxi Prov. People's Hospital, Affiliated of Shanxi Medical University, Taiyuan 030001, China.
\end{abstract}

Received: 16 October 2020 Accepted: 29 April 2021

Published online: 25 May 2021

\section{References}

1. Armstrong DK, Alvarez RD, Bakkum-Gamez JN, Barroilhet L, Behbakht K, Berchuck A, et al. NCCN guidelines insights: ovarian Cancer, version 1.2019. J Natl Compr Cancer Netw. 2019;17(8):896-909.
2. Barisic M, Aguiar P, Geley S, Maiato H. Kinetochore motors drive congression of peripheral polar chromosomes by overcoming random arm-ejection forces. Nat Cell Biol. 2014;16(12):1249-56.

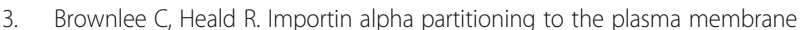
regulates intracellular scaling. Cell. 2019;176(4):805-815 e808.

4. Cai Z, Zhang MX, Tang Z, Zhang Q, Ye J, Xiong TC, et al. USP22 promotes IRF3 nuclear translocation and antiviral responses by deubiquitinating the importin protein KPNA2. J Exp Med. 2020;217(5):1-19.

5. Cao Q, Song Z, Ruan H, Wang C, Yang X, Bao L, et al. Targeting the KIF4A/ AR Axis to reverse endocrine therapy resistance in castration-resistant prostate Cancer. Clin Cancer Res. 2020;26(6):1516-28.

6. Cerami E, Gao J, Dogrusoz U, Gross BE, Sumer SO, Aksoy BA, et al. The cBio cancer genomics portal: an open platform for exploring multidimensional cancer genomics data. Cancer Discov. 2012;2(5):401-4.

7. Chandrashekar DS, Bashel B, Balasubramanya SAH, Creighton CJ, PonceRodriguez I, Chakravarthi B, et al. UALCAN: a portal for facilitating tumor subgroup gene expression and survival analyses. Neoplasia. 2017;19(8):649-58.

8. Cui X, Jing X, Wu X, Xu J, Liu Z, Huo K, et al. Analyses of DNA methylation involved in the activation of nuclear Karyopherin alpha 2 leading to identify the progression and prognostic significance across human breast Cancer. Cancer Manag Res. 2020;12:6665-77.

9. Dahl E, Kristiansen G, Gottlob K, Klaman I, Ebner E, Hinzmann B, et al. Molecular profiling of laser-microdissected matched tumor and normal breast tissue identifies karyopherin alpha2 as a potential novel prognostic marker in breast cancer. Clin Cancer Res. 2006;12(13):3950-60.

10. Force USPST, Grossman DC, Curry SJ, Owens DK, Barry MJ, Davidson KW, et al. Screening for ovarian Cancer: US preventive services task Force recommendation statement. JAMA. 2018;319(6):588-94.

11. Gao J, Aksoy BA, Dogrusoz U, Dresdner G, Gross B, Sumer SO, et al. Integrative analysis of complex cancer genomics and clinical profiles using the cBioPortal. Sci Signal. 2013;6(269):pl1.

12. Goldman MJ, Craft B, Hastie M, Repecka K, McDade F, Kamath A, et al. Visualizing and interpreting cancer genomics data via the Xena platform. Nat Biotechnol. 2020;38(6):675-8.

13. Gori S, Barberis M, Bella MA, Buttitta F, Capoluongo E, Carrera P, et al. Recommendations for the implementation of BRCA testing in ovarian cancer patients and their relatives. Crit Rev Oncol Hematol. 2019;140:67-72.

14. Hou PF, Jiang T, Chen F, Shi PC, Li HQ, Bai J, et al. KIF4A facilitates cell proliferation via induction of p21-mediated cell cycle progression and promotes metastasis in colorectal cancer. Cell Death Dis. 2018;9(5):477.

15. Hu G, Yan Z, Zhang C, Cheng M, Yan Y, Wang Y, et al. FOXM1 promotes hepatocellular carcinoma progression by regulating KIF4A expression. J Exp Clin Cancer Res. 2019;38(1):188.

16. Hua S, Ji Z, Quan Y, Zhan M, Wang H, Li W, et al. Identification of hub genes in hepatocellular carcinoma using integrated bioinformatic analysis. Aging. 2020;12(6):5439-68.

17. Jensen JB, Munksgaard PP, Sorensen CM, Fristrup N, Birkenkamp-Demtroder K, Ulhoi BP, et al. High expression of karyopherin-alpha2 defines poor prognosis in non-muscle-invasive bladder cancer and in patients with invasive bladder cancer undergoing radical cystectomy. Eur Urol. 2011;59(5):841-8.

18. Lu KH, Patterson AP, Wang L, Marquez RT, Atkinson EN, Baggerly KA, et al. Selection of potential markers for epithelial ovarian cancer with gene expression arrays and recursive descent partition analysis. Clin Cancer Res. 2004;10(10):3291-300.

19. Mizuno H, Kitada K, Nakai K, Sarai A. PrognoScan: a new database for metaanalysis of the prognostic value of genes. BMC Med Genet. 2009;2:18.

20. Mortezavi A, Hermanns T, Seifert HH, Baumgartner MK, Provenzano M, Sulser T, et al. KPNA2 expression is an independent adverse predictor of biochemical recurrence after radical prostatectomy. Clin Cancer Res. 2011;17(5):1111-21.

21. Moyer VA, Force USPST. Screening for ovarian cancer: U.S. preventive services task Force reaffirmation recommendation statement. Ann Intern Med. 2012;157(12):900-4.

22. Nagy A, Lanczky A, Menyhart O, Gyorffy B. Author correction: validation of miRNA prognostic power in hepatocellular carcinoma using expression data of independent datasets. Sci Rep. 2018;8(1):11515.

23. Niu F, Kazimierska M, Nolte IM, Terpstra MM, de Jong D, Koerts J, et al. The miR-26b-5p/KPNA2 Axis is an important regulator of Burkitt Lymphoma Cell Growth. Cancers. 2020;12(6):1464.

24. Rhodes DR, Yu J, Shanker K, Deshpande N, Varambally R, Ghosh D, et al. ONCOMINE: a cancer microarray database and integrated data-mining platform. Neoplasia. 2004;6(1):1-6. 
25. Shi C, Sun L, Liu S, Zhang E, Song Y. Overexpression of Karyopherin subunit alpha 2 (KPNA2) predicts unfavorable prognosis and promotes bladder Cancer Tumorigenicity via the P53 pathway. Med Sci Monit. 2020;26: e921087.

26. Tang Z, Li C, Kang B, Gao G, Li C, Zhang Z. GEPIA: a web server for cancer and normal gene expression profiling and interactive analyses. Nucleic Acids Res. 2017;45(W1):W98-W102.

27. Taniwaki M, Takano A, Ishikawa N, Yasui W, Inai K, Nishimura H, et al. Activation of KIF4A as a prognostic biomarker and therapeutic target for lung cancer. Clin Cancer Res. 2007;13(22 Pt 1):6624-31.

28. Vaughan S, Coward JI, Bast RC Jr, Berchuck A, Berek JS, Brenton JD, et al. Rethinking ovarian cancer: recommendations for improving outcomes. Nat Rev Cancer. 2011;11(10):719-25.

29. Welsh JB, Zarrinkar PP, Sapinoso LM, Kern SG, Behling CA, Monk BJ, et al. Analysis of gene expression profiles in normal and neoplastic ovarian tissue samples identifies candidate molecular markers of epithelial ovarian cancer. Proc Natl Acad Sci U S A. 2001;98(3):1176-81.

30. Winnepenninckx V, Lazar V, Michiels S, Dessen P, Stas M, Alonso SR, et al. Gene expression profiling of primary cutaneous melanoma and clinical outcome. J Natl Cancer Inst. 2006;98(7):472-82.

31. Wright AA, Bohlke K, Armstrong DK, Bookman MA, Cliby WA, Coleman RL, et al. Neoadjuvant chemotherapy for newly diagnosed, advanced ovarian Cancer: Society of Gynecologic Oncology and American Society of clinical oncology clinical practice guideline. J Clin Oncol. 2016;34(28):3460-73.

32. Wu G, Chen PL. Structural requirements of chromokinesin Kif4A for its proper function in mitosis. Biochem Biophys Res Commun. 2008;372(3):454-8.

33. Yang F, Li S, Cheng Y, Li J, Han X. Karyopherin alpha 2 promotes proliferation, migration and invasion through activating NF-kappaB/p65 signaling pathways in melanoma cells. Life Sci. 2020;252:117611.

34. Zhang L, He M, Zhu W, Lv X, Zhao Y, Yan Y, et al. Identification of a panel of mitotic spindle-related genes as a signature predicting survival in lung adenocarcinoma. J Cell Physiol. 2020;235(5):4361-75.

\section{Publisher's Note}

Springer Nature remains neutral with regard to jurisdictional claims in published maps and institutional affiliations.

Ready to submit your research? Choose BMC and benefit from:

- fast, convenient online submission

- thorough peer review by experienced researchers in your field

- rapid publication on acceptance

- support for research data, including large and complex data types

- gold Open Access which fosters wider collaboration and increased citations

- maximum visibility for your research: over $100 \mathrm{M}$ website views per year

At BMC, research is always in progress.

Learn more biomedcentral.com/submissions 